\title{
Measurement of high energy dark matter from the Sun at IceCube
}

\section{Ye Xu}

School of Electronic, Electrical Engineering and Physics, Fujian University of Technology, Fuzhou 350118, China

Research center for Microelectronics Technology, Fujian University of Technology, Fuzhou 350118, China

E-mail: xuy@fjut.edu.cn

ABSTRACT: It is assumed that heavy dark matter particles (HDMs) with a mass of $\mathrm{O}(\mathrm{TeV})$ are captured by the Sun. HDMs can decay to relativistic light dark matter particles (LDMs), which could be measured by $\mathrm{km}^{3}$ neutrino telescopes (like the IceCube detector). The numbers and fluxes of expected LDMs and neutrinos were evaluated at IceCube with the $Z^{\prime}$ portal dark matter model. Based on the assumption that no events are observed at IceCube in 6 years, the corresponding upper limits on LDM fluxes were calculated at $90 \%$ C. L.. These results indicated that LDMs could be directly detected in the $\mathrm{O}(1 \mathrm{TeV})$ $\mathrm{O}(10 \mathrm{TeV})$ energy range at IceCube with $100 \mathrm{GeV} \lesssim m_{Z^{\prime}} \lesssim 350 \mathrm{GeV}$ and $\tau_{\phi} \lesssim 5 \times 10^{22} \dot{\mathrm{s}}$.

Keywords: Dark matter, Dark Matter and Double Beta Decay (experiments)

ARXIV EPRINT: 2106.02202 


\section{Contents}

1 Introduction 1

2 HDM accumulation in the Sun 2

3 LDM and neutrino interactions with nuclei 3

4 Flux of LDMs which reach the Earth 4

5 Evaluation of the numbers of expected LDMs and neutrinos at IceCube 4

$\begin{array}{llr}6 & \text { Results } & 6\end{array}$

$\begin{array}{llr}7 & \text { Discussion and conclusion } & 8\end{array}$

\section{Introduction}

It was found in cosmological and astrophysical observations that the bulk of matter in the Universe consists of dark matter (DM). $84 \%$ of the matter content is thermal DM in the Universe, which were created thermally in Early Universe [1-3]. The searches for high energy (from $\mathrm{O}(\mathrm{GeV})$ to $\mathrm{O}(\mathrm{TeV})$ ) neutrinos which are produced by the DM annihilation in the Sun's core have been performed using the data recorded by the IceCube and ANTARES neutrino telescopes $[4,5]$. But no one has found thermal DM particles yet [4-9].

The heavy dark sector with a mass of $\mathrm{O}(\mathrm{TeV})$ is an alternative DM scenario [10-20]. In this model, there exist at least two DM species in the Universe (for example, heavy and light DM particles). Heavy dark matter (HDM), $\phi$, is a thermal particle which is generated by the early universe. The bulk of present-day DM consists of them. The other is a stable light dark matter particle (LDM), $\chi$ which is the product of the decay of HDM $(\phi \rightarrow \chi \bar{\chi})$. Due to the decay of long-living HDMs $\left(\tau_{\phi} \gg t_{0}[21,22], t_{0}\right.$ is the age of the Universe), the present-day DM may also contain a small component which is high energy LDMs. Besides direct measurements of HDMs, one can detect the standard model (SM) products of decay of HDMs. The search for these high energy SM particles from the Sun (they should be neutrinos in this measurement) has been performed using the data recorded by the IceCube neutrino observatory [23]. In this work, however, the products of the decay of HDMs are a class of LDMs [24, 25], not SM particles.

The LDMs from the Sun's core could be more easily detected with IceCube, compared to those from the Earth's core, since the HDM accumulation in the Sun is much greater than that in the Earth [26, 27]. LDMs would interact with nuclei when they pass through the Sun, the Earth and ice. Those LDMs can be directly measured with the IceCube neutrino telescope. The capability of the measurement of those LDMs will be discussed 
here. In this measurement, the background consists of muons and neutrinos generated in cosmic ray interactions in the Earth's atmosphere and astrophysical neutrinos.

In what follows, the distributions and numbers of expected LDMs and neutrinos will be evaluated in the energy range $1-100 \mathrm{TeV}$ assuming 6 years of IceCube data. Then the upper limits on LDM fluxes were also calculated at 90\% C.L.. Finally, the capability of the measurement of $\mathrm{TeV}$ LDMs will be evaluated at IceCube.

\section{HDM accumulation in the Sun}

HDMs of the Galactic halo would collide with atomic nuclei in the Sun when their wind sweeps through the Sun. A fraction of those HDMs would lose enough kinetic energy to be trapped in orbit. With further collisions with atomic nuclei in the Sun's interior, they would eventually thermalize and settle in the Sun's core under the influence of gravitation of the Sun's interior. Those HDMs inside the Sun can decay into LDMs at an appreciable rate. Then the number $\mathrm{N}$ of HDMs, captured by the Sun, is obtained by the following equation [26]

$$
\frac{d N}{d t}=C_{\text {cap }}-2 \Gamma_{\text {ann }}-C_{\text {evp }} N-C_{\text {dec }} N
$$

where $C_{\text {cap }}, \Gamma_{\text {ann }}$ and $C_{\text {evp }}$ are the capture rate, the annihilation rate and the evaporation rate, respectively. The evaporation rate is only relevant when the DM mass $<5 \mathrm{GeV}$ [26], which are much lower than my interested mass scale (the mass of HDM, $\mathrm{m}_{\phi} \geq 1 \mathrm{TeV}$ ). Thus their evaporation contributes to the accumulation in the Sun at a negligible level in the present work. $C_{\text {dec }}$ is the decay rate for HDMs. Since the fraction of HDM decay $\leq$ $3.2 \times 10^{-14}$ per year $\left(\tau_{\phi} \geq 10^{21} \mathrm{~s}\right)$, its contribution to the HDM accumulation in the Sun can be ignored in the evaluation of HDM accumulation. $\Gamma_{\text {ann }}$ is obtained by the following equation [26]

$$
\Gamma_{\text {ann }}=\frac{C_{\text {cap }}}{2} \tanh ^{2}\left(\frac{t}{\tau}\right) \approx \frac{C_{\text {cap }}}{2} \quad \text { with } \quad t \gg \tau
$$

where $\tau=\left(C_{\text {cap }} C_{\text {ann }}\right)^{-\frac{1}{2}}$ is a time-scale set by the competing processes of capture and annihilation. At late times $t \gg \tau$ one can approximate $\tanh ^{2} \frac{t}{\tau}=1$ in the case of the Sun [26]. $C_{\text {cap }}$ is proportional to $\frac{\sigma_{\phi N}}{m_{\phi}}[26,30]$, where $m_{\phi}$ is the mass of HDM and $\sigma_{\phi N}$ is the scattering cross section between the nucleons and HDMs. The spin-independent cross section is only considered in the capture rate calculation. Then $\sigma_{\phi N}$ is taken to be $10^{-44}$ $\mathrm{cm}^{2}$ for $m_{\phi} \sim \mathrm{O}(\mathrm{TeV})[6,7]$.

The HDM distribution in the Sun is obtained by [26],

$$
n(r)=n_{0} \exp \left(-\frac{r^{2}}{r_{\phi}^{2}}\right), \text { with } r_{\phi}=\left(\frac{3 T_{s}}{2 \pi G_{N} \rho_{s} m_{\phi}}\right)^{1 / 2} \approx 0.01 R_{\text {sun }} \sqrt{\frac{100 T e V}{m_{\phi}}}
$$

where $\mathrm{G}_{N}$ is the Newtonian gravitational constant. $\rho_{s} \approx 151 \mathrm{~g} / \mathrm{cm}^{3}$ and $T_{s} \approx 15.5 \times 10^{6} \mathrm{~K}$ are the matter density and temperature at the sun center, respectively. $\mathrm{R}_{\text {sun }}$ is the radius of the Sun. One finds that HDMs are concentrated around the center of the Sun. 


\section{$3 \quad$ LDM and neutrino interactions with nuclei}

In this work, a $Z^{\prime}$ portal dark matter model $[28,29]$ is taken for LDMs to interact with nuclei via a neutral current interaction mediated by a gauge boson $Z^{\prime}$ which couples to both the LDMs and quarks (see figure 1a in ref. [24]). Here a LDM is assumed to be a Dirac fermion. As assumed in ref. [24], the interaction vertexes $\left(\chi \chi Z^{\prime}\right.$ and $\left.q q Z^{\prime}\right)$ are vector-like in this model, since $Z^{\prime}$ vector boson typically acquires mass through the breaking of an additional U(1) gauge group at high energies. This deep inelastic scattering (DIS) crosssection for $\chi+N \rightarrow \chi+$ anything ( $N$ is a nucleus) is computed in the same way as the ref. [24] in this work. The effective interaction Lagrangian can be written as follows:

$$
\mathcal{L}=\bar{\chi} g_{\chi \chi Z^{\prime}} \gamma^{\mu} \chi Z_{\mu}^{\prime}+\sum_{q_{i}} \bar{q}_{i} g_{q q Z^{\prime}} \gamma^{\mu} q_{i} Z_{\mu}^{\prime}
$$

where $q_{i}$ 's are the SM quarks, and $g_{\chi \chi} Z^{\prime}$ and $g_{q q Z^{\prime}}$ are the $Z^{\prime}-\chi$ and $Z^{\prime}-q_{i}$ couplings, respectively. This Deep inelastic scattering (DIS) cross-section is computed in the labframe using tree-level CT10 parton distribution functions [31]. The coupling constant G $\left(G=g_{\chi \chi} Z^{\prime} g_{q q Z^{\prime}}\right)$ is chosen to be 0.05 . The masses of $Z^{\prime}$ are taken to be $100 \mathrm{GeV}$, and $250 \mathrm{GeV}$ and $350 \mathrm{GeV}$, respectively. Here the mass of LDM $m_{\chi}$ is assumed to be $8 \mathrm{GeV}$, then the outgoing energy of LDM caused by the decay of HDM $\mathrm{E}_{\chi} \approx \frac{1}{2} m_{\phi}$. The computed DIS cross section obeys a simple power-law form for the energies between $1 \mathrm{TeV}$ and $1 \mathrm{PeV}$. With $\mathrm{m}_{Z^{\prime}}=250 \mathrm{GeV}$, for example, its cross section is obtained by the following function:

$$
\sigma_{\chi N}=1.284 \times 10^{-41} \mathrm{~cm}^{2}\left(\frac{E_{\chi}}{1 G e V}\right)^{0.970}
$$

where $\mathrm{E}_{\chi}$ is the LDM energy.

The DIS cross-section for neutrino interaction with nuclei is computed in the lab-frame and given by simple power-law forms [32] for neutrino energies above $1 \mathrm{TeV}$ :

$$
\begin{gathered}
\sigma_{\nu N}(C C)=4.74 \times 10^{-35} \mathrm{~cm}^{2}\left(\frac{E_{\nu}}{1 G e V}\right)^{0.251} \\
\sigma_{\nu N}(N C)=1.80 \times 10^{-35} \mathrm{~cm}^{2}\left(\frac{E_{\nu}}{1 G e V}\right)^{0.256}
\end{gathered}
$$

where $\sigma_{\nu N}(C C)$ and $\sigma_{\nu N}(N C)$ are the DIS cross-sections for neutrino interaction with nuclei via a charge current $(\mathrm{CC})$ and neutral current $(\mathrm{NC})$, respectively. $E_{\nu}$ is the neutrino energy.

The inelasticity parameter $y=1-\frac{E_{\chi^{\prime}, \text { lepton }}}{E_{\text {in }}}$ (where $E_{\text {in }}$ is the incoming LDM or neutrino energy and $E_{\chi^{\prime}}$,lepton is the outgoing DM particles or lepton energy). $E_{s e c}=y E_{\text {in }}$, where $E_{\text {sec }}$ is the secondaries' energy after a LDM or neutrino interaction with nuclei. The mean values of $y$ for LDMs have been computed:

$$
\langle y\rangle=\frac{1}{\sigma\left(E_{\mathrm{in}}\right)} \int_{0}^{1} y \frac{d \sigma}{d y}\left(E_{\mathrm{in}}, y\right) d y
$$

The LDM and neutrino interaction lengths can be obtained by

$$
L_{\nu, \chi}=\frac{1}{N_{A} \rho \sigma_{\nu, \chi N}}
$$


where $N_{A}$ is the Avogadro constant, and $\rho$ is the density of matter, which LDMs and neutrinos interact with.

\section{Flux of LDMs which reach the Earth}

The LDMs which reach the Earth are produced by the decay of HDMs in the Sun's core. These LDMs have to pass through the Sun and interact with nuclei inside the Sun. Then the number $\mathrm{N}_{s}$ of LDMs which reach the Sun's surface is obtained by the following equation:

$$
\begin{aligned}
N_{s} & =2 N_{0}\left(\exp \left(-\frac{t_{0}}{\tau_{\phi}}\right)-\exp \left(-\frac{t_{0}+T}{\tau_{\phi}}\right)\right) \prod_{i=1}^{n=\mathcal{N}} \exp \left(\frac{\delta L}{L_{i}}\right) \quad \text { with } T \ll \tau_{\phi} \\
& \approx 2 N_{0} \frac{T}{\tau_{\phi}} \exp \left(-\frac{t_{0}}{\tau_{\phi}}\right) \prod_{i=1}^{n=\mathcal{N}} \exp \left(\frac{\delta L}{L_{i}}\right)
\end{aligned}
$$

where $\mathrm{N}_{0}=\int_{0}^{t_{s}} \frac{d N}{d t} d t$ is the number of HDMs captured in the Sun. $\mathrm{t}_{s}$ and $\mathrm{t}_{0}$ are the ages of the Sun and the Universe, respectively. $\mathrm{T}$ is the lifetime of taking data for IceCube and taken to be 6 years. If the distance from the Sun's center to the Sun's surface is equally divided into $\mathcal{N}$ portions, $\delta L=\frac{R_{\mathrm{sun}}}{\mathcal{N}} . L_{i}=\frac{1}{N_{A} \rho_{i} \sigma_{\chi N}}$ is the LDM interaction length at $\mathrm{i} \times \delta L$ away from the Sun's center. $\rho_{i}$ is the density at $i \times \delta L$ away from the Sun's center [33]. $\mathrm{N}_{s}$ is computed in column density in the present work. The first exponential term in the eq. (4.1) is the fraction of decay of HDMs in the Sun's core. The term of continued product in the eq. (4.1) is the faction of LDMs which reach the Sun's surface. Here $\mathcal{N}$ is taken to be $10^{4}$. The results with $\mathcal{N}=10^{4}$ is sufficiently accurate, whose uncertainty is about $0.05 \%$.

Then the flux $\Phi_{\text {LDM }}$ of LDMs, which reach the Earth, from the Sun's core is described by

$$
\Phi_{\mathrm{LDM}}=\frac{N_{s}}{4 \pi D_{s e}^{2}}
$$

where $D_{s e}$ is the distance between the Sun and Earth.

\section{Evaluation of the numbers of expected LDMs and neutrinos at IceCube}

The lifetime for HDMs decaying into SM particles is strongly constrained $\left(\tau \geq \mathrm{O}\left(10^{26}\right.\right.$ $\left.10^{29}\right) \mathrm{s}$ ) by diffuse gamma and neutrino observations [22, 34-36]. Since the present work considers an assumption that HDMs are unable to decay to SM particles, the constraints on the lifetime for HDM are only those based on cosmology (the age of the Universe is about $10^{17}$ $\mathrm{s})$. Since $\tau_{\phi} \gg 10^{17} \mathrm{~s}$ in the $Z^{\prime}$ portal dark matter model [21, 22], $\tau_{\phi} \geq 10^{21} \mathrm{~s}$ in this work.

IceCube is a $\mathrm{km}^{3}$ neutrino telescope and deployed in the deep ice below the geographic South Pole [37]. It can detect neutrino interactions with nuclei via the measurement of the cascades caused by their secondary particles above the energy threshold of $1 \mathrm{TeV}$ [38]. The LDMs from the Sun, which pass through the IceCube detector, would interact with the nuclei inside IceCube. This is very similar to the DIS of neutrino interaction with nuclei via a neutral current, whose secondary particles would develop into a cascade at IceCube.

In this analysis, LDM events were selected with the following event selection criteria. First, only cascade events were kept. The track-like events are a class of background sources. 
The track-like events initiated by muons due to atmospheric muons and muon neutrinos would be rejected after that event selection. To reduce more background events initiated by atmospheric muon, Second, only up-going events occurring during a period in which the Sun was below the horizon were kept. Besides, only those up-going events from the Sun's direction were kept. Due to the sizable energy and angular uncertainties caused by the event reconstruction with IceCube, the cut windows for energy and angular separation between cascades and the Sun's direction would be used to extract signal candidate events from the up-going cascades events. These windows were taken to be one standard uncertainty and one median uncertainty, respectively. Certainly, the residual signals still contain a small neutrino component after all those event selections. Since the LDM and neutrino cascades are hard to distinguish at IceCube, one could only evaluate the number of expected neutrinos fallen into those windows.

The factors $\left(C_{1}\right.$ and $\left.C_{2}\right)$ should be considered in the evaluation of the numbers of expected LDMs. $C_{1}$ is equal to $68.3 \%$ (that is $68.3 \%$ of the LDM events reconstructed with IceCube fall into a window caused by one standard energy uncertainty). $C_{2}$ is equal to $50 \%$ (that is $50 \%$ of the LDM events reconstructed with IceCube fall into a window caused by one median angular uncertainty). Then the number $\mathrm{N}_{\text {det }}$ of expected LDMs obeys the following equation:

$$
\frac{d N_{\mathrm{det}}}{d E}=C_{1} \times C_{2} \times \int_{T} A_{\mathrm{eff}}(E) \Phi_{\mathrm{LDM}} P(E, \epsilon(t)) d t
$$

where $A_{\text {eff }}(E)$ obtained from the figure 2 in ref. [39] is denoting the effective observational area for IceCube. $\mathrm{E}$ is denoting the energy of an incoming particle. $P(E, \epsilon(t))$ can be given by the following equation:

$$
P(E, \epsilon(t))=\exp \left(-\frac{D_{e}(\epsilon(t))}{L_{\text {earth }}}\right)\left(1-\exp \left(-\frac{D}{L_{\text {ice }}}\right)\right) .
$$

where $L_{\text {earth,ice }}$ is denoting the LDM interaction lengths with the Earth and ice, respectively. $\mathrm{D}$ is denoting the effective length in the IceCube detector and taken to be $1 \mathrm{~km}$ in this work. $D_{e}(\epsilon(t))=2 R_{e} \sin (\epsilon(t))$ is denoting the distance through the Earth. $\mathrm{R}_{e}$ is denoting the radius of the Earth. $\epsilon(t)$ is denoting the obliquity of the ecliptic changing with time. The maximum value of $\epsilon$ is $23.44^{\circ}$.

After rejecting track-like events, the background remains two sources: astrophysical and atmospheric neutrinos which pass through the detector of IceCube. Only a neural current interaction with nuclei is relevant to muon neutrinos considered here. The astrophysical neutrinos flux can be described by [40]

$$
\Phi_{\nu}^{\text {astro }}=\Phi_{\text {astro }} \times\left(\frac{E_{\nu}}{100 T e V}\right)^{-\left(\alpha+\beta \log _{10}\left(\frac{E_{\nu}}{100 T e V}\right)\right)} \times 10^{-18} \mathrm{GeV}^{-1} \mathrm{~cm}^{-2} s^{-1} s r^{-1}
$$

where $\Phi_{\nu}^{\text {astro }}$ is denoting the total astrophysical neutrino flux. The coefficients, $\Phi_{\text {astro }}, \alpha$ and $\beta$ are given in figure VI.10 in ref. [40]. The atmospheric neutrinos flux can be described by $[41]$

$$
\Phi_{\nu}^{\mathrm{atm}}=C_{\nu}\left(\frac{E_{\nu}}{1 G e V}\right)^{-\left(\gamma_{0}+\gamma_{1} x+\gamma_{2} x^{2}\right)} G e V^{-1} c m^{-2} s^{-1} s r^{-1}
$$




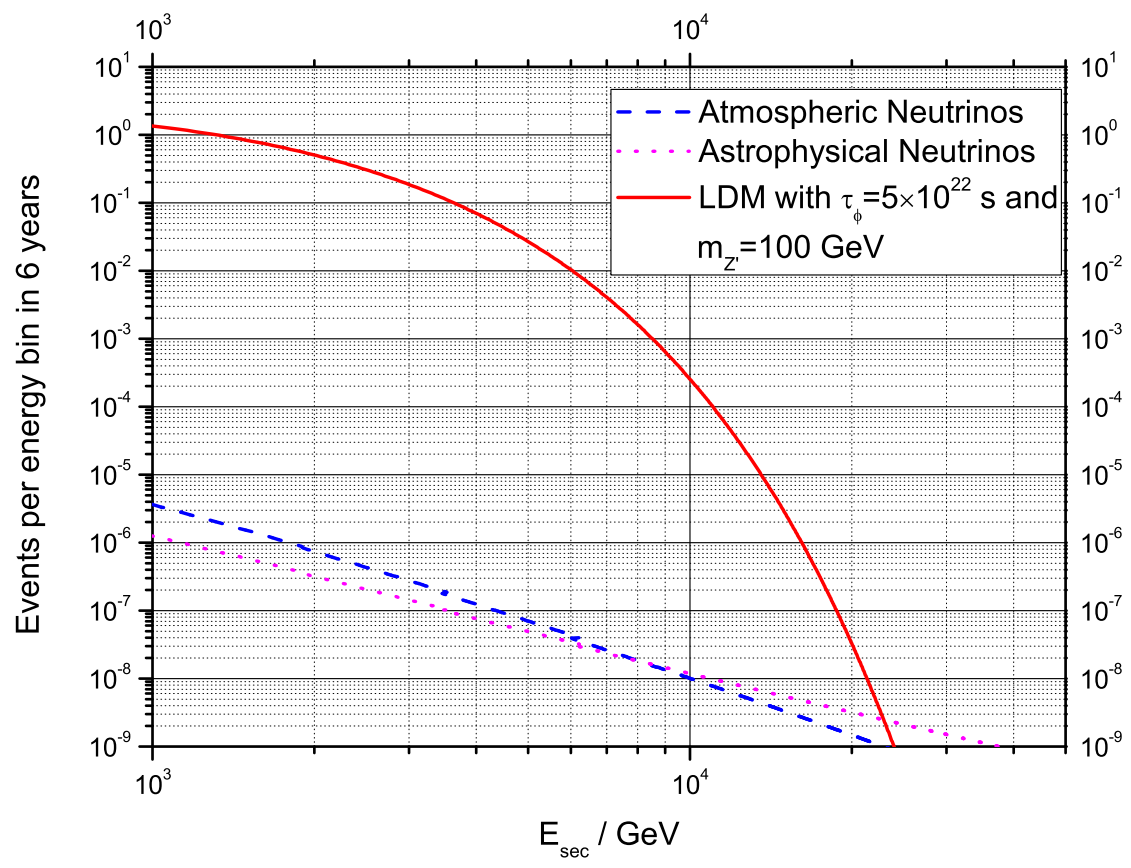

Figure 1. Distributions of expected LDMs with $\tau_{\phi}=5 \times 10^{22} \mathrm{~s}$ and $m_{Z^{\prime}}=100 \mathrm{GeV}$ and neutrinos. Their energy bins are $100 \mathrm{GeV}$.

where $x=\log _{10}\left(E_{\nu} / 1 G e V\right) . \Phi_{\nu}^{\text {atm }}$ is denoting the atmospheric neutrino flux. The coefficients, $C_{\nu}\left(\gamma_{0}, \gamma_{1}\right.$ and $\left.\gamma_{2}\right)$ are given in table III in ref. [41].

The neutrinos fallen into the energy and angular windows mentioned above would also be regarded as signal candidate events, so the evaluation of the number of expected neutrinos has to be performed by integrating over the region caused by these windows. Then the number of expected neutrinos $\mathrm{N}_{\nu}$ obeys the following equation:

$$
\frac{d N_{\nu}}{d E}=\int_{T} \int_{\theta_{\min }}^{\theta_{\max }} A_{\mathrm{eff}}(E)\left(\Phi_{\nu}^{\mathrm{astro}}+\Phi_{\nu}^{\mathrm{atm}}\right) P(E, \epsilon(t), \theta) \frac{2 \pi r_{e}(\epsilon(t))^{2} \sin 2 \theta}{D_{e}^{\prime}(\epsilon(t), \theta)^{2}} d \theta d t
$$

where $r_{e}(\epsilon(t))=\frac{D_{e}(\epsilon(t))}{2}$. $\theta$ is denoting the angular separation between the neutrinos and the Sun's diretion. $\theta_{\min }=0$ and $\theta_{\max }=\sigma_{\theta} . \sigma_{\theta}$ is denoting the median angular uncertainty for cascades at IceCube. The standard energy and median angular uncertainties can be obtained from ref. [42] and ref. [43], respectively. $P(E, \epsilon(t), \theta)$ can be given by

$$
P(E, \epsilon(t), \theta)=\exp \left(-\frac{D_{e}^{\prime}(\epsilon(t), \theta)}{L_{\text {earth }}}\right)\left(1-\exp \left(-\frac{D}{L_{\text {ice }}}\right)\right)
$$

where $D_{e}^{\prime}(\epsilon(t), \theta)=D_{e}(\epsilon(t)) \cos (\theta)$ is denoting the distance through the Earth.

\section{$6 \quad$ Results}

The distributions and numbers of expected LDMs and neutrinos were evaluated in the energy range $1-100 \mathrm{TeV}$ assuming 6 years of IceCube data. Figure 1 shows the distributions 


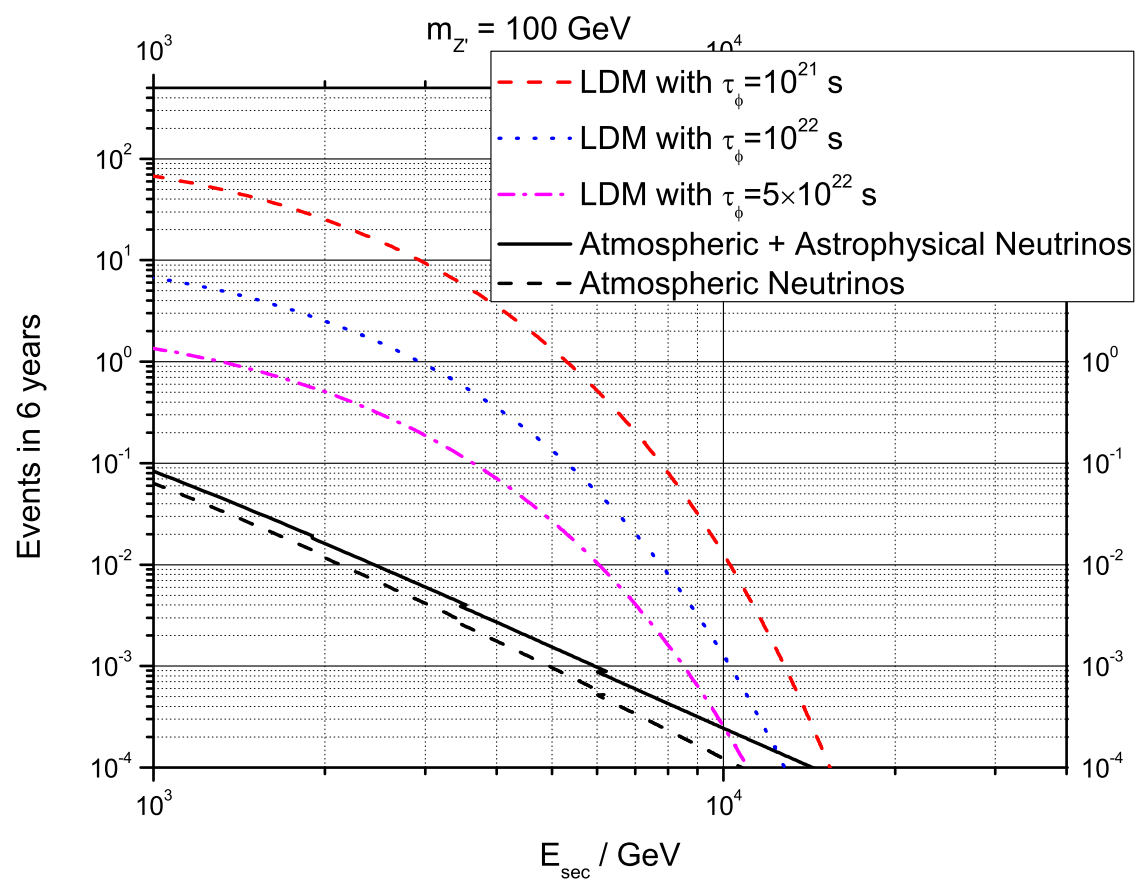

Figure 2. With the different $\tau_{\phi}\left(10^{21} \mathrm{~s}, 10^{22} \mathrm{~s}\right.$ and $\left.5 \times 10^{22} \mathrm{~s}\right)$, the numbers of expected LDMs were evaluated assuming 6 years of IceCube data, respectively. The evaluation of numbers of expected neutrinos was performed by integrating over the regions caused by one standard energy and median angular uncertainties.

(with an energy bin of $100 \mathrm{GeV}$ ) of expected LDMs and neutrinos. Compared to LDMs with $m_{Z^{\prime}}=100 \mathrm{GeV}$ and $\tau_{\phi}=5 \times 10^{22} \mathrm{~s}$, the numbers of neutrino events per energy bin are at least smaller by 4 orders of magnitude in the energy range $1-10 \mathrm{TeV}$. As shown in figure 1 , the dominant background is caused by atmospheric neutrinos in the energy range $1-5 \mathrm{TeV}$ but astrophysical neutrinos at energies above about $10 \mathrm{TeV}$ in this measurement.

The numbers of expected neutrinos (see black solid line) are shown in figure 2 and 3. The evaluation of the numbers of expected neutrinos was performed by integrating over the region caused by the energy and angular windows described above. The black dash line denotes the number of expected atmospheric neutrinos. These two figures both indicate the neutrino background can be ignored in this measurement. The numbers of expected LDMs with $m_{Z^{\prime}}=100 \mathrm{GeV}$ and $\tau_{\phi}=10^{21} \mathrm{~s}$ can reach about 70 and 1 at $1 \mathrm{TeV}$ and $5.3 \mathrm{TeV}$ at IceCube, respectively, as shown in figure 2 (see the red dash line). Figure 2 also presents LDMs with $\tau_{\phi}=10^{22} \mathrm{~s}$ (see the blue dot line) and $\tau_{\phi}=5 \times 10^{22} \mathrm{~s}$ (see the magenta dash dot line) could be detected below about $3 \mathrm{TeV}$ and $1.3 \mathrm{TeV}$ at IceCube, respectively, when $m_{Z^{\prime}}=100 \mathrm{GeV}$. Figure 3 presents LDMs with $m_{Z^{\prime}}=250 \mathrm{GeV}$ (see the blue dot line) and $m_{Z^{\prime}}=350 \mathrm{GeV}$ (see the magenta dash dot line) could be detected below about $36 \mathrm{TeV}$ and $4 \mathrm{TeV}$ at IceCube, respectively, when $\tau_{\phi}=10^{21} \mathrm{~s}$. 


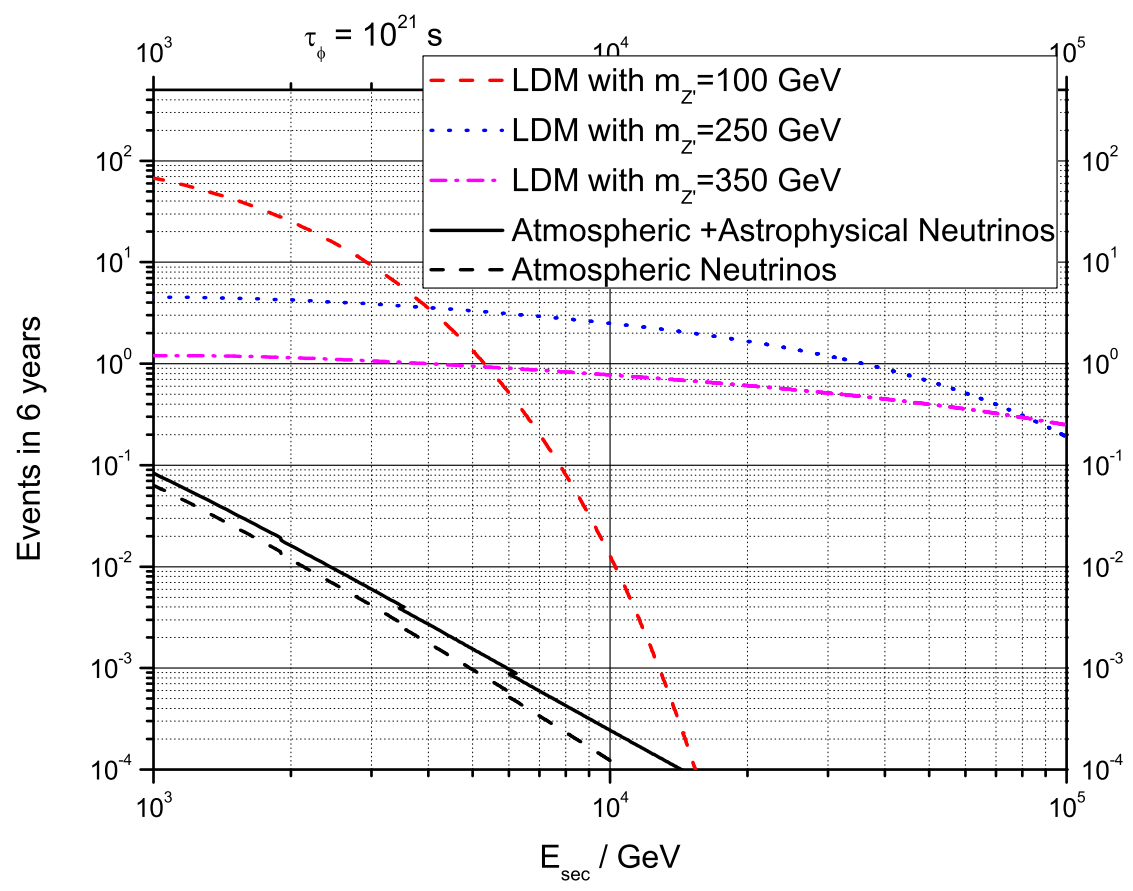

Figure 3. With the different $Z^{\prime}$ masses $(100 \mathrm{GeV}, 250 \mathrm{GeV}$ and $350 \mathrm{GeV})$, the numbers of expected LDMs were evaluated assuming 6 years of IceCube data, respectively. The evaluation of numbers of expected neutrinos was performed by integrating over the regions caused by one standard energy and median angular uncertainties.

\section{Discussion and conclusion}

The ref. [44] presents an analysis of neutrino signals due to the DM annihilation in the Sun with 6 years of IceCube data. This analysis has not found any significant indication of neutrinos due to the DM annihilation in the Sun. Since the LDM and neutrino signals are hard to distinguish at IceCube, it is a reasonable assumption that no events are observed in the measurement of LDMs due to the decay of HDM in the Sun at IceCube in 6 years. The corresponding upper limit on LDM flux at 90\% C.L. was calculated with the FeldmanCousins approach [45] (see the black solid line in figure 4 and 5).

Figure 4 presents the fluxes of expected LDM with $\tau_{\phi}=10^{21} \mathrm{~s}$ (red dash line), $10^{22} \mathrm{~s}$ (blue dot line) and $5 \times 10^{22} \mathrm{~s}$ (magenta dash dot line). That limit excludes the LDM fluxes with $\tau_{\phi}=10^{21} \mathrm{~s}$ and $10^{22} \mathrm{~s}$ below about $4.4 \mathrm{TeV}$ and $2.1 \mathrm{TeV}$, respectively. Then the LDMs could be probed with $\tau_{\phi} \lesssim 5 \times 10^{22}$ s at IceCube.

Figure 5 shows the fluxes of expected LDM with $m_{Z^{\prime}}=100 \mathrm{GeV}$ (red dash line), $250 \mathrm{GeV}$ (blue dot line) and $350 \mathrm{GeV}$ (magenta dash dot line). That limit excludes the LDM fluxes with $m_{Z^{\prime}}=100 \mathrm{GeV}$ and $250 \mathrm{GeV}$ below about $4.4 \mathrm{TeV}$ and $10.3 \mathrm{TeV}$, respectively. Then the LDMs could be probed with $100 \mathrm{GeV} \lesssim m_{Z^{\prime}} \lesssim 350 \mathrm{GeV}$ at IceCube.

Based on the results described above, it is a reasonable conclusion that those LDMs could be directly detected in the $\mathrm{O}(1 \mathrm{TeV})-\mathrm{O}(10 \mathrm{TeV})$ energy range at IceCube with $100 \mathrm{GeV}$ 


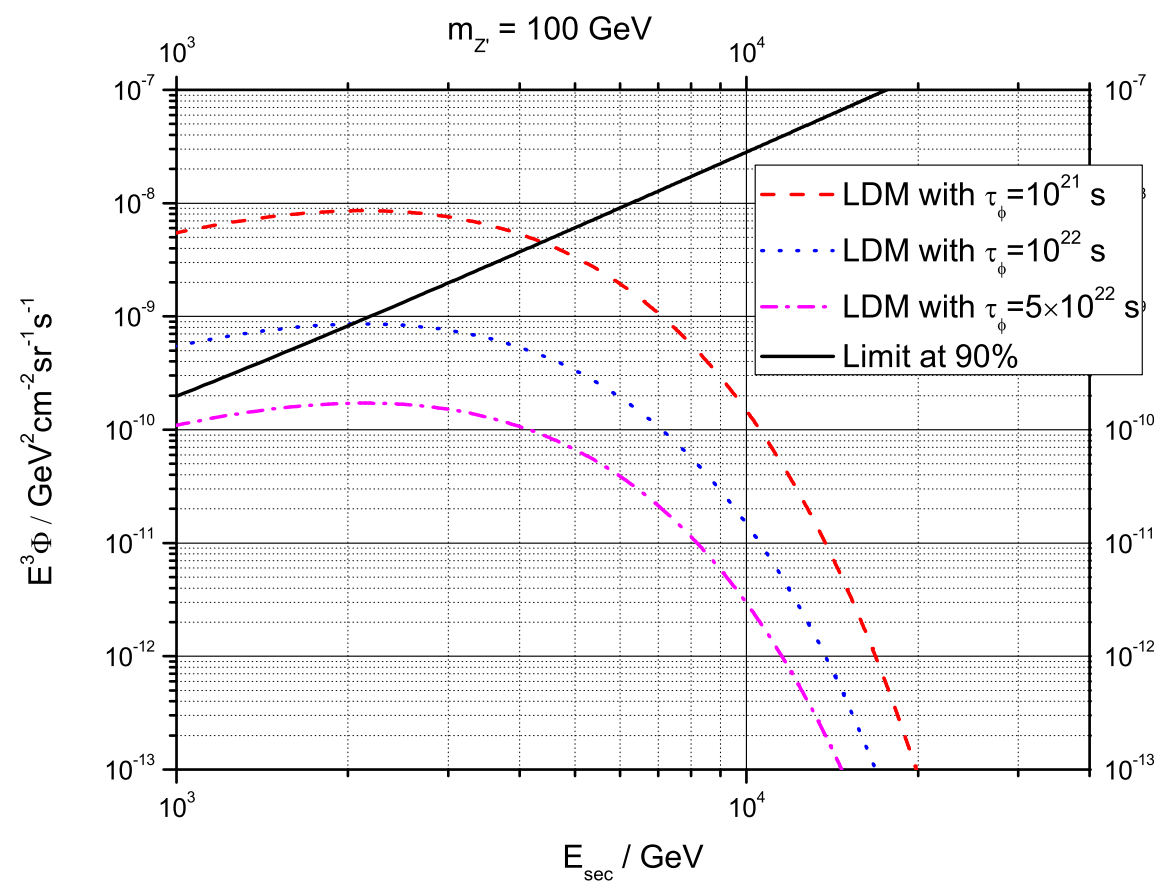

Figure 4. With the different $\tau_{\phi}\left(10^{21} \mathrm{~s}, 10^{22} \mathrm{~s}\right.$ and $\left.5 \times 10^{22} \mathrm{~s}\right)$, the fluxes of expected LDM were estimated at IceCube, respectively. Assuming no observation at IceCube in 6 years, the upper limit at $90 \%$ C.L. was also computed.

$\lesssim m_{Z^{\prime}} \lesssim 350 \mathrm{GeV}$ and $\tau_{\phi} \lesssim 5 \times 10^{22}$ s. Since these constraints are only given by the assumptions mentioned above, certainly, the experimental collaborations, like the IceCube collaboration, should be encouraged to conduct an unbiased analysis with the data of IceCube.

Since $\Phi_{\mathrm{LDM}}$ is proportional to $\frac{1}{\tau_{\phi}}$ (see eq. (4.1)), the above results actually depends on the lifetime of heavy DM, $\tau_{\phi}$. If $\tau_{\phi}$ varies from $10^{18} \mathrm{~s}$ to $10^{20} \mathrm{~s}$, the numbers of expected LDMs with IceCube are larger by from 3 to 1 orders of magnitude than that with $\tau_{\phi}=10^{21}$ s, respectively.

Besides, the capability of the measurement of those LDMs was roughly evaluated with the ANTARES telescope. Those LDMs could be directly detected at energies with $\mathrm{O}(1 \mathrm{TeV})$ at ANTARES with $m_{Z^{\prime}}<200 \mathrm{GeV}$ and $\tau_{\phi}<10^{20} \mathrm{~s}$. Compared to IceCube, the expected signal to background rate is larger by about one order of magnitude with ANTARES. It is more difficult for ANTARES to detect those LDMs, however, since the effective area for ANTARES is smaller by about 2 orders of magnitude than that for IceCube at energies above $1 \mathrm{TeV}[39,46]$. As we all know, the capabilities of the measurement of those LDMs should be substantially improved with IceCube and ANTARES if their upgrading projects will be completed in the future.

Ref. [28] presents an analysis of the constraints on the mass of $Z^{\prime}$ and $g_{\chi \chi} Z^{\prime}$ using the observations of direct, indirect measurement, collider and cosmology. Considered the assumption that the bulk of present-day DM consists of HDMs in this work, observations 


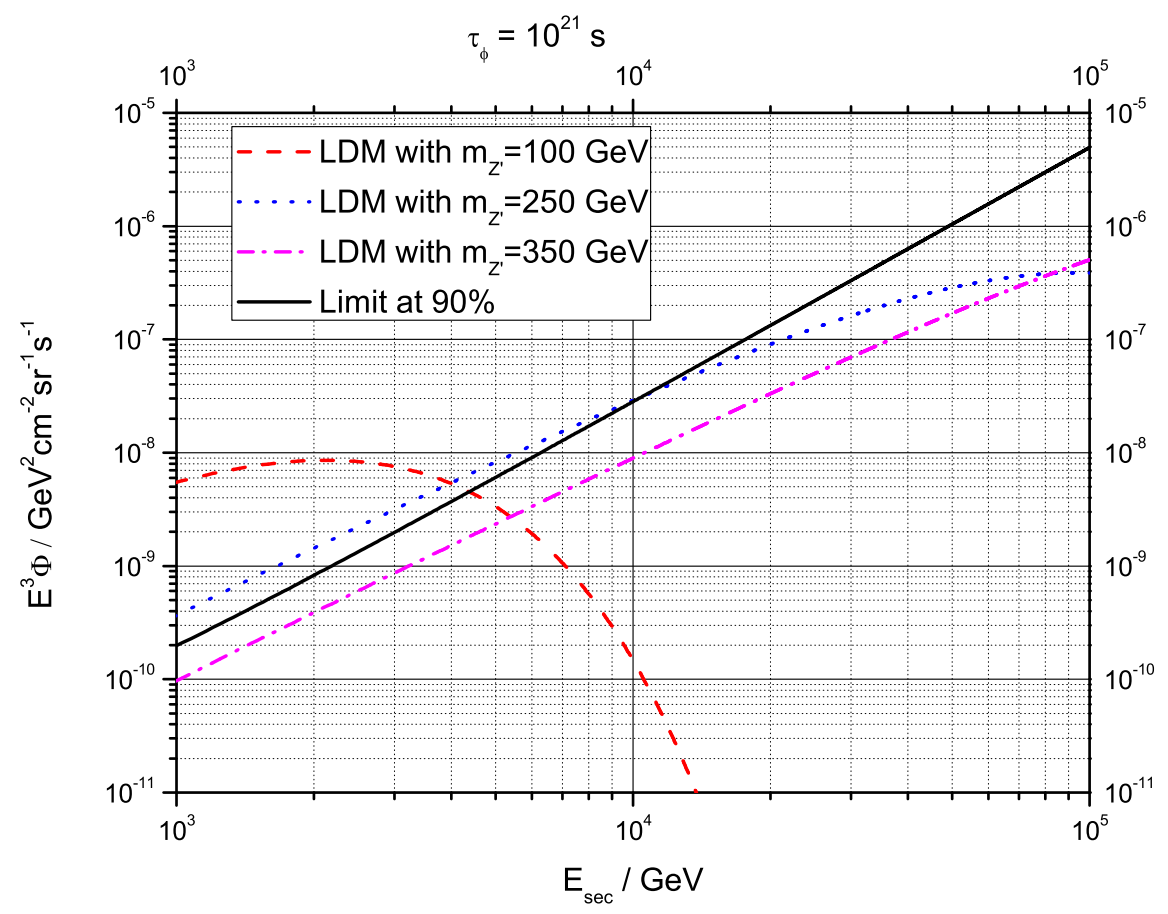

Figure 5. With the different $Z^{\prime}$ masses $(100 \mathrm{GeV}, 250 \mathrm{GeV}$ and $350 \mathrm{GeV})$, the fluxes of expected LDM were estimated at IceCube, respectively. Assuming no observation at IceCube in 6 years, the upper limit at $90 \%$ C.L. was also computed.

of cosmology and direct, indirect measurements of DM are inappropriate to analysis the results in this work. Figure 6 in ref. [28] shows the result for DM with a mass of $8 \mathrm{GeV}$. The whole light $Z^{\prime}$ window $\left(\mathrm{m}_{Z^{\prime}}<1 \mathrm{TeV}\right)$ is ruled out by the observations of LHC and Tevatron using the dijet data. To measure DM generated by colliders, the dijet+ $\mathrm{E}_{T}^{\text {miss }}$ analysis is more reasonable. Figure 6 in ref. [28] presents the light $Z^{\prime}$ window is not ruled out by $\mathrm{LHC}$ at $8 \mathrm{TeV}$ using the dijet $+\mathrm{E}_{T}^{\text {miss }}$ data with $g_{\chi \chi Z^{\prime}}<0.25$.

\section{Acknowledgments}

This work was supported by the National Natural Science Foundation of China (NSFC) under the contract No. 11235006, the Science Fund of Fujian University of Technology under the contracts No. GY-Z14061 and GY-Z13114 and the Natural Science Foundation of Fujian Province in China under the contract No. 2015J01577.

Open Access. This article is distributed under the terms of the Creative Commons Attribution License (CC-BY 4.0), which permits any use, distribution and reproduction in any medium, provided the original author(s) and source are credited. 


\section{References}

[1] L. Bergström, Nonbaryonic dark matter: observational evidence and detection methods, Rept. Prog. Phys. 63 (2000) 793 [hep-ph/0002126] [InSPIRE].

[2] G. Bertone, D. Hooper and J. Silk, Particle dark matter: evidence, candidates and constraints, Phys. Rept. 405 (2005) 279 [hep-ph/0404175] [INSPIRE].

[3] Planck collaboration, Planck 2015 results. XIII. Cosmological parameters, Astron. Astrophys. 594 (2016) A13 [arXiv:1502.01589] [INSPIRE].

[4] ICECuBE collaboration, Search for annihilating dark matter in the Sun with 3 years of IceCube data, Eur. Phys. J. C 77 (2017) 146 [Erratum ibid. 79 (2019) 214] [arXiv: 1612.05949] [INSPIRE].

[5] ANTARES collaboration, Limits on dark matter annihilation in the Sun using the ANTARES neutrino telescope, Phys. Lett. B 759 (2016) 69 [arXiv: 1603.02228] [InSPIRE].

[6] XENON collaboration, First dark matter search results from the XENON1T experiment, Phys. Rev. Lett. 119 (2017) 181301 [arXiv:1705.06655] [InSPIRE].

[7] PandaX-II collaboration, Dark matter results from 54-ton-day exposure of PandaX-II experiment, Phys. Rev. Lett. 119 (2017) 181302 [arXiv:1708.06917] [InSPIRE].

[8] FERMI-LAT collaboration, Limits on dark matter annihilation signals from the Fermi LAT 4-year measurement of the isotropic gamma-ray background, JCAP 09 (2015) 008 [arXiv: 1501.05464] [INSPIRE].

[9] ANTARES and ICECUBE collaborations, Combined search for neutrinos from dark matter self-annihilation in the galactic center with ANTARES and IceCube, Phys. Rev. D 102 (2020) 082002 [arXiv:2003.06614] [INSPIRE].

[10] M.Yu. Khlopov and V.M. Chechetkin, Antiprotons in the universe as a cosmological test of grand unification, Sov. J. Part. Nucl. 18 (1987) 267.

[11] D.J.H. Chung, E.W. Kolb and A. Riotto, Nonthermal supermassive dark matter, Phys. Rev. Lett. 81 (1998) 4048 [hep-ph/9805473] [INSPIRE].

[12] D.J.H. Chung, E.W. Kolb and A. Riotto, Superheavy dark matter, Phys. Rev. D 59 (1998) 023501 [hep-ph/9802238] [InSPIRE].

[13] V. Kuzmin and I. Tkachev, Ultrahigh-energy cosmic rays, superheavy long living particles, and matter creation after inflation, JETP Lett. 68 (1998) 271 [Pisma Zh. Eksp. Teor. Fiz. 68 (1998) 255] [hep-ph/9802304] [INSPIRE].

[14] E.W. Kolb, D.J.H. Chung and A. Riotto, WIMPzillas!, AIP Conf. Proc. 484 (1999) 91 [hep-ph/9810361] [INSPIRE].

[15] D.J.H. Chung, E.W. Kolb, A. Riotto and I.I. Tkachev, Probing Planckian physics: resonant production of particles during inflation and features in the primordial power spectrum, Phys. Rev. D 62 (2000) 043508 [hep-ph/9910437] [INSPIRE].

[16] D.J.H. Chung, P. Crotty, E.W. Kolb and A. Riotto, On the gravitational production of superheavy dark matter, Phys. Rev. D 64 (2001) 043503 [hep-ph/0104100] [INSPIRE].

[17] E.W. Kolb, A.A. Starobinsky and I.I. Tkachev, Trans-Planckian wimpzillas, JCAP 07 (2007) 005 [hep-th/0702143] [INSPIRE]. 
[18] L. Covi, M. Grefe, A. Ibarra and D. Tran, Neutrino signals from dark matter decay, JCAP 04 (2010) 017 [arXiv:0912.3521] [INSPIRE].

[19] B. Feldstein, A. Kusenko, S. Matsumoto and T.T. Yanagida, Neutrinos at IceCube from heavy decaying dark matter, Phys. Rev. D 88 (2013) 015004 [arXiv:1303.7320] [InSPIRE].

[20] M.A. Fedderke, E.W. Kolb and M. Wyman, Irruption of massive particle species during inflation, Phys. Rev. D 91 (2015) 063505 [arXiv: 1409.1584] [INSPIRE].

[21] R. Aloisio, S. Matarrese and A.V. Olinto, Super heavy dark matter in light of BICEP2, Planck and ultra high energy cosmic rays observations, JCAP 08 (2015) 024 [arXiv: 1504.01319] [INSPIRE].

[22] A. Esmaili, A. Ibarra and O.L.G. Peres, Probing the stability of superheavy dark matter particles with high-energy neutrinos, JCAP 11 (2012) 034 [arXiv:1205.5281] [INSPIRE].

[23] ICECuBE collaboration, Search for neutrinos from decaying dark matter with IceCube, Eur. Phys. J. C 78 (2018) 831 [arXiv: 1804.03848] [INSPIRE].

[24] A. Bhattacharya, R. Gandhi and A. Gupta, The direct detection of boosted dark matter at high energies and PeV events at IceCube, JCAP 03 (2015) 027 [arXiv: 1407.3280] [InSPIRE].

[25] A. Bhattacharya, R. Gandhi, A. Gupta and S. Mukhopadhyay, Boosted Dark Matter and its implications for the features in IceCube HESE data, JCAP 05 (2017) 002 [arXiv: 1612.02834] [INSPIRE].

[26] P. Baratella, M. Cirelli, A. Hektor, J. Pata, M. P IIBeleht and A. Strumia, PPPC $4 D M \nu$ : a Poor Particle Physicist Cookbook for neutrinos from Dark Matter annihilations in the Sun, JCAP 03 (2014) 053 [arXiv: 1312.6408] [INSPIRE].

[27] J. Lundberg and J. Edsjo, WIMP diffusion in the solar system including solar depletion and its effect on earth capture rates, Phys. Rev. D 69 (2004) 123505 [astro-ph/0401113] [INSPIRE].

[28] A. Alves, S. Profumo and F.S. Queiroz, The dark $Z^{\prime}$ portal: direct, indirect and collider searches, JHEP 04 (2014) 063 [arXiv: 1312.5281] [INSPIRE].

[29] D. Hooper, $Z^{\prime}$ mediated dark matter models for the galactic center gamma-ray excess, Phys. Rev. D 91 (2015) 035025 [arXiv:1411.4079] [INSPIRE].

[30] G. Jungman, M. Kamionkowski and K. Griest, Supersymmetric dark matter, Phys. Rept. 267 (1996) 195 [hep-ph/9506380] [INSPIRE].

[31] H.-L. Lai et al., New parton distributions for collider physics, Phys. Rev. D 82 (2010) 074024 [arXiv: 1007.2241] [INSPIRE].

[32] M.M. Block, P. Ha and D.W. McKay, Ultrahigh energy neutrino scattering: an update, Phys. Rev. D 82 (2010) 077302 [arXiv: 1008.4555] [INSPIRE].

[33] R.F. Stein and A. Nordlund, Simulations of solar granulation. I. General properties, Astrophys. J. 499 (1998) 914.

[34] K. Murase and J.F. Beacom, Constraining very heavy dark matter using diffuse backgrounds of neutrinos and cascaded gamma rays, JCAP 10 (2012) 043 [arXiv:1206.2595] [INSPIRE].

[35] C. Rott, K. Kohri and S.C. Park, Superheavy dark matter and IceCube neutrino signals: bounds on decaying dark matter, Phys. Rev. D 92 (2015) 023529 [arXiv:1408.4575] [INSPIRE]. 
[36] M. Kachelriess, O.E. Kalashev and M.Y. Kuznetsov, Heavy decaying dark matter and IceCube high energy neutrinos, Phys. Rev. D 98 (2018) 083016 [arXiv: 1805.04500] [INSPIRE].

[37] IceCube collaboration, Sensitivity of the IceCube detector to astrophysical sources of high energy muon neutrinos, Astropart. Phys. 20 (2004) 507 [astro-ph/0305196] [INSPIRE].

[38] ICECubE collaboration, Search for sources of astrophysical neutrinos using seven years of IceCube cascade events, Astrophys. J. 886 (2019) 12 [arXiv:1907.06714] [INSPIRE].

[39] ICECUBE collaboration, Searches for extended and point-like neutrino sources with four years of IceCube data, Astrophys. J. 796 (2014) 109 [arXiv:1406.6757] [INSPIRE].

[40] ICECuBE collaboration, The IceCube high-energy starting event sample: description and flux characterization with 7.5 years of data, Phys. Rev. D 104 (2021) 022002 [arXiv:2011.03545] [INSPIRE].

[41] T.S. Sinegovskaya, A.D. Morozova and S.I. Sinegovsky, High-energy neutrino fluxes and flavor ratio in the Earth's atmosphere, Phys. Rev. D 91 (2015) 063011 [arXiv:1407.3591] [INSPIRE].

[42] ICECuBE collaboration, Energy reconstruction methods in the IceCube neutrino telescope, 2014 JINST 9 P03009 [arXiv:1311.4767] [INSPIRE].

[43] ICECUBE collaboration, Search for astrophysical sources of neutrinos using cascade events in IceCube, Astrophys. J. 846 (2017) 136 [arXiv:1705. 02383] [INSPIRE].

[44] ICECuBE collaboration, Search for secluded dark matter with 6 years of IceCube data, PoS ICRC2021 (2021) 521 [arXiv:2107.10778] [INSPIRE].

[45] G.J. Feldman and R.D. Cousins, A unified approach to the classical statistical analysis of small signals, Phys. Rev. D 57 (1998) 3873 [physics/9711021] [INSPIRE].

[46] ANTARES and ICECUBE collaborations, The first combined search for neutrino point-sources in the southern hemisphere with the Antares and IceCube neutrino telescopes, Astrophys. J. 823 (2016) 65 [arXiv:1511.02149] [INSPIRE]. 